\title{
GESTIÓN DE OBJETOS DE APRENDIZAJE MEDIANTE SISTEMATIZACIÓN DE PROCESOS
}

\section{LEARNING OBJECTS MANAGEMENT BY PROCESS SYSTEMATIZATION}

\author{
Elio Cárdenas Alas², Janeth Herrera Rojas', Miguel Fernando Inga Avila
}

\begin{abstract}
RESUMEN
En la investigación se identifican los problemas relacionados con la generación, ensamblaje y reutilización de los objetos de aprendizaje. En particular, se ha identificado la carencia de modelos conceptuales en el diseño y construcción de los actuales sistemas para la gestión del contenido del aprendizaje. Estos sistemas, aunque permiten en su mayoría, la integración y reutilización de objetos de aprendizaje, no proporcionan mecanismos de ensamblaje que respeten el conocimiento asociado (requisitos y competencias) a los objetos de aprendizaje. Además, no se define un procedimiento para la descripción de los objetos resultantes de dichas integraciones mediante el uso de meta-datos (información acerca de información). Por lo anterior se propone: (i) un modelo conceptual que proporciona las pautas para facilitar la generación, ensamblaje y reutilización de objetos de aprendizaje. Los objetos de aprendizaje generados con éste modelo reciben el nombre de ELOs) y se definen como recursos educativos descritos por meta-datos y organizados en una estructura multicapa, cuyos elementos más avanzados incluyen conocimiento asociado; (ii) un mecanismo, basado en ontologías, para el ensamblaje de diferentes tipos de ELOs, (iii) un procedimiento para la descripción, mediante metadatos, de los ELOs resultantes del proceso de ensamblaje y (iv) un prototipo de la herramienta Deacon que implementa el marco conceptual propuesto en el presente trabajo.
\end{abstract}

Palabras clave: Objetos de aprendizaje, conocimiento asociado, elearning, unidad de información, unidad de contenido, unidad didáctica, meta-datos, granularidad

\begin{abstract}
In the investigation we identify the problems related to the generation, assembly and reuse of learning objects, in particular, the lack of conceptual models for designing and building learning content management systems. Although the majority of these systems allow integration and reuse of learning objects, they do not provide assembly mechanisms which take into account the knowledge (requirements and competencies) associated to the learning objects (Associated Knowledge). Moreover, no procedure exists to produce meta-data descriptions of the objects resulting from such integration. That before we propose: (i) a conceptual model, which provides guidelines to facilitate generation, assembly and reuse of learning objects. We call the learning objects generating with this model Electronic Learning Objects. An educational resource described by meta-data and organized in a multilayer structure, in which higher-level elements posses associated knowledge; (ii) a mechanism, based on ontology, for the assembly of different types of learning objects, (iii) a procedure for producing a meta-data description of ELOs resulting from the assembly processes., (iv) a prototype of deacon tool that implements the propose of conceptual frame in the present work.
\end{abstract}

Key words: Learning objects, associated knowledge, e-Learning, information unit, content unit, didactic unit, meta-data, granularity

\footnotetext{
${ }^{2}$ Universidad Nacional del Centro del Perú. Facultad de Ingeniería de Sistemas-Estudiantes.

${ }^{3}$ Universidad Nacional del Centro del Perú. Facultad de Ingeniería de Sistemas.
} 


\section{INTRODUCCIÓN}

Si bien las innovaciones tecnológicas representan importantes factores de cambio, dentro del contexto educativo ese efecto de cambio ha estado enmarcado por los rápidos desarrollos en aspectos como el acceso, la formación continua y el eLearning. Las especificaciones y herramientas actuales son neutrales en términos pedagógicos, de contexto y de formato, lo que desde el punto de vista técnico, a corto plazo, favorece la reutilización y la interoperabilidad. Sin embargo, para los profesores dichas especificaciones y herramientas reutilizable; cada $\mathrm{UI}$ contiene un único archivo multimedia que el usuario puede localizar, recuperar y manipular separadamente.

Unidades de Contenido (UCs): Se encuentra en el Nivel 1 de la estructura multicapa. Una UC se forma ensamblando Uls, o ensamblando Uls y UCs. Estas representan una experiencia educativa con conocimiento asociado, es decir, que una UC necesita de unos requisitos para su comprensión y proporcionan unas competencias tras su comprensión.

carecen de valor pedagógico, puesto que los factores que para ellos son fundamentales, como el hecho de poder utilizar modelos educativos, estilos de aprendizaje, entre otros, han sido en algunos casos eliminados.

Esto ha motivado la promoción del uso de tecnologías como los objetos de aprendizaje, también han trascendido al contexto de la industria, en el que los esfuerzos para el desarrollo de contenidos de

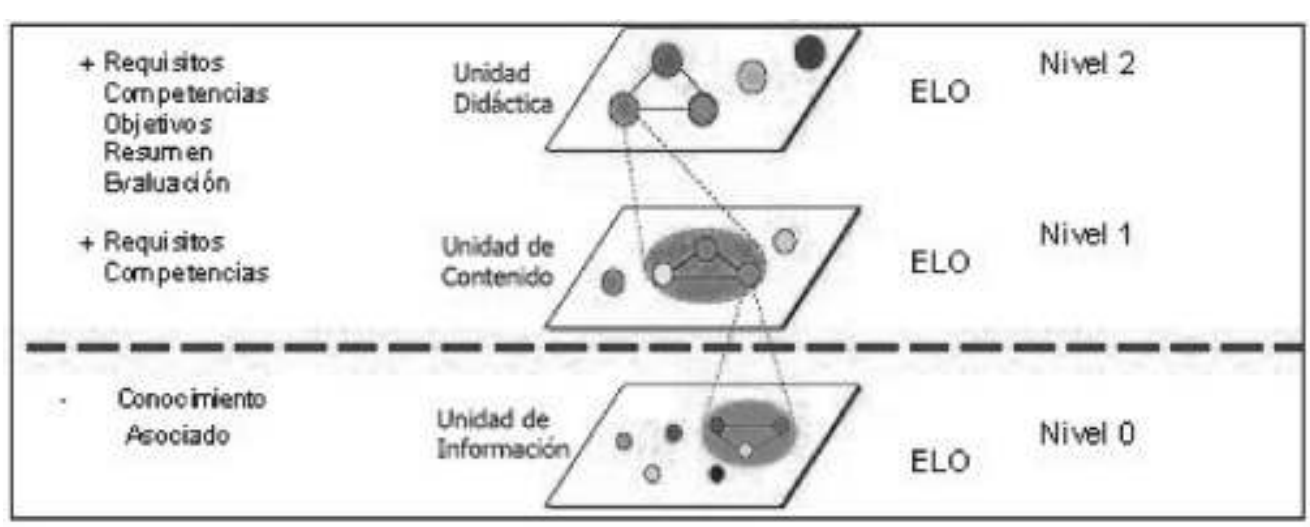

Figura 1. Niveles de granularidad.

\section{Elaboración propia.}

aprendizaje deberían beneficiarse de piezas de contenido integrables, que se pudieran reensamblar según las necesidades de diseño. Las limitaciones se deben a que las herramientas no están orientadas a las necesidades educativas; requieren que el usuario sea un experto en las tecnologías XML; no proporcionan mecanismos para el ensamblaje de nuevos objetos de aprendizaje en los que se respete el conocimiento asociado a dichos objetos; y tampoco los sistemas de e-Learning, como los LCMSs (Learning Content Management Systems), definen un modelo conceptual para la generación, ensamblaje y reutilización de objetos de aprendizaje.

La investigación tuvo como objetivo fundamental proporcionar un modelo conceptual para la generación, ensamblaje y reutilización de objetos de aprendizaje.

\section{MATERIAL Y METODOS}

El modelo conceptual propuesto para la generación, ensamblaje y reutilización de objetos de aprendizaje, consta de un modelo de contenido cuyos componentes son:

Unidades de Información (UIs): Se encuentran en el Nivel 0. Una UI es un elemento atómico autocontenido y por lo tanto, altamente

Unidades Didácticas (UDs): se encuentra en el Nivel 2 de la estructura multicapa. Se forma ensamblando UCs, UCs con UDs, o UDs. Una UD representa el conocimiento referente a un área tras la acumulación de diversas experiencias relacionadas. Al igual que las UCs una UD incluye conocimiento asociado.

Los elementos contenidos en cada uno de estos niveles determinan el grado de componibilidad de los objetos de aprendizaje, el cual depende del conocimiento asociado a cada elemento. Como se muestra en la Figura 1.

Marco conceptual propuesto: Ensamblaje de ELOs: El proceso de ensamblaje proporciona una forma consistente para relacionar diferentes tipos de componentes del modelo de contenido ELO, mediante la aplicación de un mecanismo de ensamblaje, de tal forma que se respete el

Tabla 1. Resultado de ensamblar ELOs pertenecientes a distintos niveles de granularidad.

\begin{tabular}{lccc}
\hline & UI (Nivel 0) & UC (Nivel 1) & UD (Nwel 2) \\
\hline UI (Nivel 0) & UC & UC & X \\
UC (Nwel 1) & UC & UD & UD \\
UD (Nivel 2) & $X$ & UD & UD \\
\hline
\end{tabular}

Elaboración propia. 
Conocimiento Asociado.

La Tabla 1, muestra las posibles combinaciones que se pueden presentar entre los elementos pertenecientes a los distintos niveles de granularidad (Figura 1).

\section{Mecanismo de ensamblaje basado en:}

- Ontologías: descripción de conceptos y relaciones que pueden existir para un usuario o una comunidad de usuarios. que se sabe que una competencia (o conjunto) cubre a un requisito (o conjunto) se puede aplicar la regla para el cálculo del conocimiento asociado del ELO ensamblado.

\section{RESULTADOS}

Los resultados están orientados a la implementación del modelo conceptual para la generación, ensamblaje y reutilización de objetos de aprendizaje, propuesto y descrito.

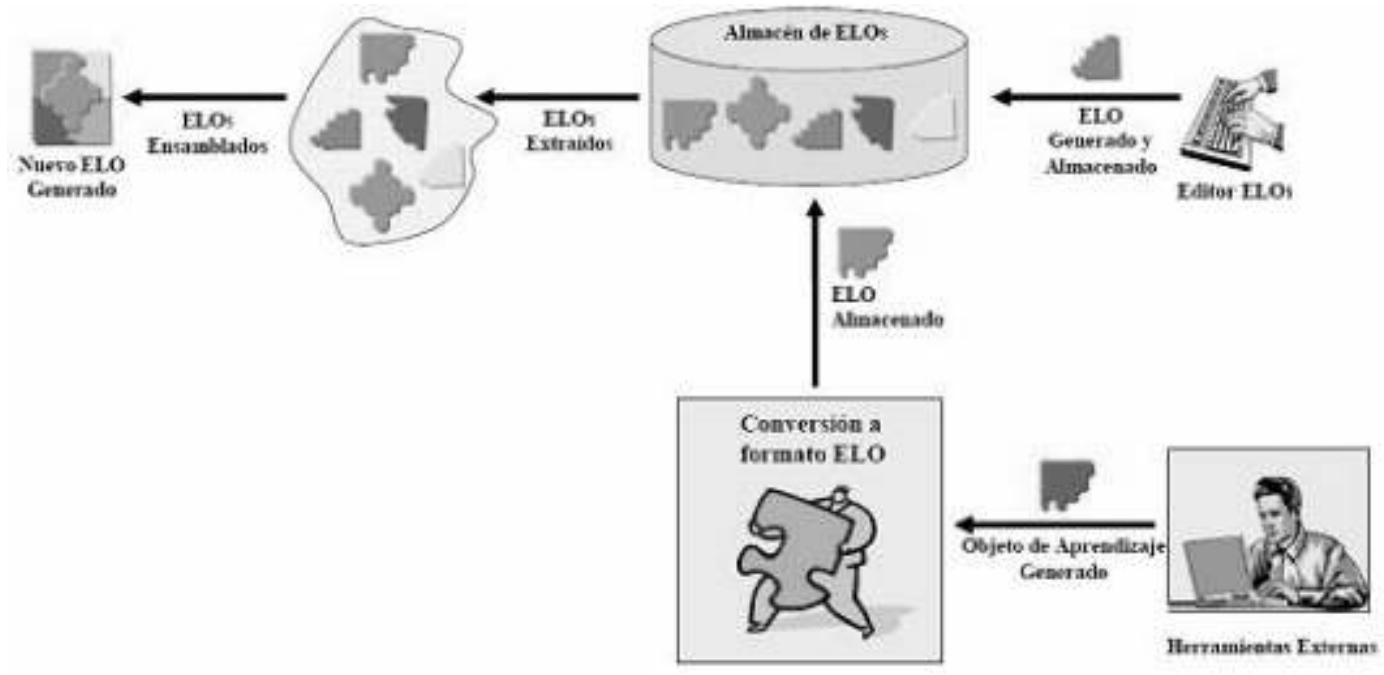

Figura 2. Funciones de Deacon.

Fuente: Elaboración propia.

La herramienta Deacon es una plataforma para el soporte de los p r o c e s o s d e generación, ensamblaje y reutilización de objetos de aprendizaje, y su importancia dentro de este proceso de evaluación radica en que permite verificar la factibilidad técnica del modelo conceptual propuesto. La Figura 2, re presenta las funciones realizadas por Deacon. En ella se observa que los ELOs son almacenados en el almacén de ELOs mediante dos procesos.

Funciones (mappings) entre ontologías: proceso por el cual dos ontologías están relacionadas a nivel conceptual, y los individuos de la ontología origen se transforman en entidades en la ontología destino de acuerdo con las relaciones semánticas establecidas.

- Dos versiones del mecanismo:

- Ontojoin: basado en ontologías que permite comparar semánticamente los requisitos y las competencias pertenecientes a ELOs heterogéneos.

- Ontojoin Full: fuerza la existencia de una ontología en la que tanto $D$ como $C$ tenga una representación.

Con independencia de usar Figura 3. Composición de la herramienta Deacan.

OntoJoin u OntoJoin Full, una vez

Fuente: Elaboración propia.

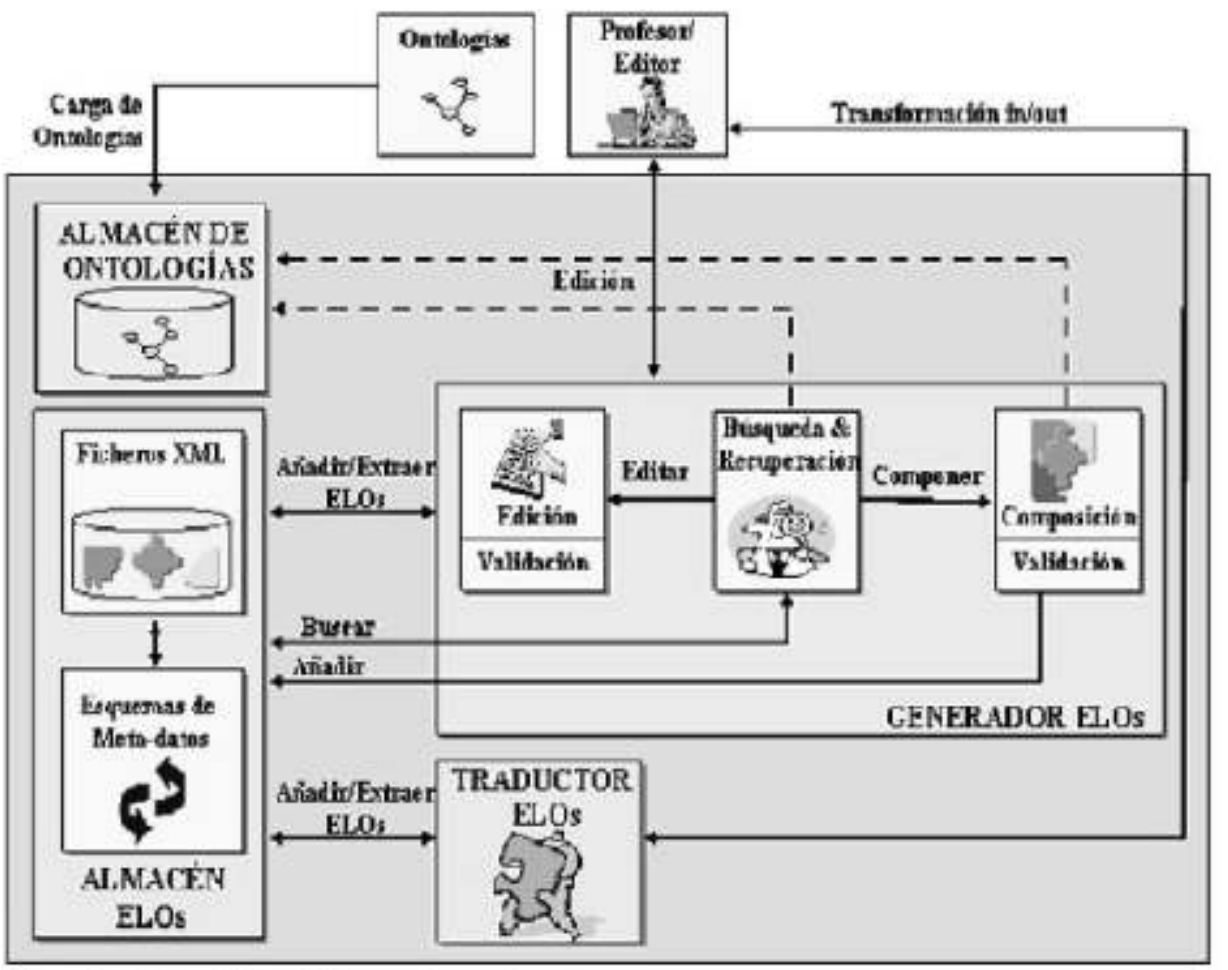


El primero, obteniendo los objetos de aprendizaje desde herramientas externas, en cuyo caso deben ser convertidos al formato ELO, es decir descritos utilizando meta-datos LOM (en su forma genérica, si se trata de UIs, o extendida, si se trata de UCS y/o UDs). El segundo, dentro del propio entorno Deacon, ya que los ELOs pueden ser recuperados desde el almacén de ELOs, con lo cual la generación de nuevos ELOs (UCS o UDS), se realiza a través del ensamblaje de ELOs ya existentes en el propio almacén.

Esta descripción permite identificar las operaciones básicas que realiza Deacon:

- Almacenar ELOs Deacon permite almacenar ELOs que, como se ha comentado anteriormente, pueden ser generados dentro de la propia herramienta o provenir de herramientas externas, en cuyo caso deben haber pasado por un proceso de traducción a formato ELO.

- Editar ELOs la herramienta permite manipular (editar, borrar, etc.) los ELOs para reutilizarlos o ensamblarlos para generar nuevos ELOs.

- Convertir (traducir) los formatos de meta-datos. Deacon permite convertir los meta-datos que describen a los objetos de aprendizaje provenientes de herramientas externas, al formato de meta-datos ELO con el fin de poder almacenarlos para su posterior reutilización.

- Ensamblar ELOs heterogéneos. Deacon implementa el mecanismo de ensamblaje basado en ontologías OntoJoin Full, a través del cual se determina si dos ELOs pueden o no ser ensamblados para generar nuevos ELOs.
En las figuras 3 y 4 , se describen la composición, modularidad e Interface de la herramienta Deacon, que le permite ejecutar las funciones mencionadas.

\section{DISCUSIÓN}

Respecto a los objetos de aprendizaje, se ha podido constatar que representan una pieza clave en el proceso de desarrollo de material didáctico, dentro de los nuevos escenarios de elearning. Sin embargo, se han observado algunos problemas relacionados con los siguientes aspectos: la falta de consenso en la propia definición de lo que es un objeto de aprendizaje.

Los modelos de contenido existentes, no proporcionan una descripción formal de sus componentes. La reutilización de objetos de aprendizaje, exige la adecuada utilización de los meta-datos. La definición de un conjunto de reglas de composición que regulen la construcción de los nuevos objetos de aprendizaje a partir de los ya existentes.

Además, no existen propuestas en las que se utilicen las características del propio objeto de aprendizaje, como medio para la definición de mecanismos que permitan el ensamblaje de diferentes tipos de objetos de aprendizaje.

Respecto a los meta-datos, se ha observado que, los actuales esquemas de meta-datos carecen de elementos adecuados que permitan describir el Conocimiento Asociado a los objetos de aprendizaje, haciendo necesario añadir extensiones a dichos esquemas.

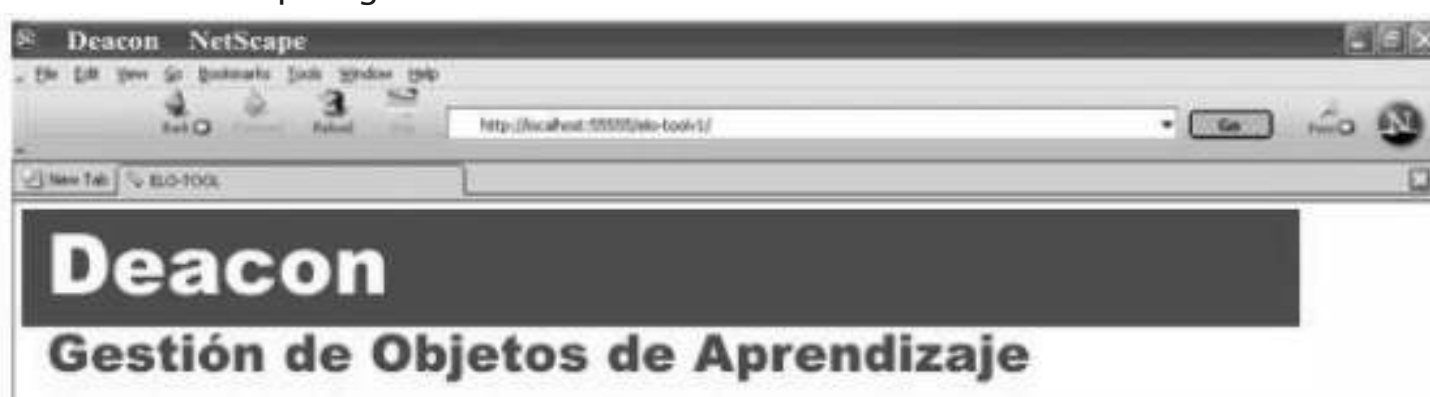

-Gestion de Ontologias

- Gestion de ELOS

- Traduccion de LOS

- Ensamblaje de Elos

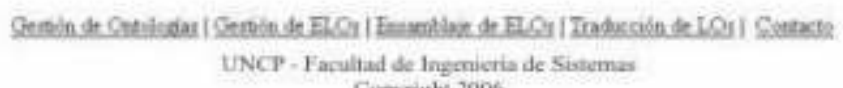

Figura 4. Interface de la herramienta Deacon.

Fuente: Elaboración propia.
Las bue n a s descripciones de o b je to s d e a prendizaje $m$ e d i a $n$ t e metadatos, facilitan los procesos de b ú s q u e d a y recuperación de los mismos y además potencian la reutilización de dichos objetos de aprendizaje. No existen tácticas, que posibiliten la obtención del conjunto de metadatos que describan a un objeto de a prend i z a je resultante de un proceso d e 
ensamblaje. Queda aún por resolver muchas preguntas, como: ¿cuál es el mejor esquema de meta-datos que debe aplicarse para cubrir las necesidades del creador, los almacenes de recursos y sus usuarios?, ¿cómo capitalizar su utilización para anticiparse a futuras aplicaciones?, y ¿qué estrategias se deben utilizar para asegurar el mantenimiento de los meta-datos de acuerdo con las versiones más recientes de los esquemas aplicados?

\section{REFERENCIAS BILBLIOGRÁFICAS}

1. Santacruz L P, Aedo I, Delgado C. Objetos de aprendizaje: Tendencias dentro de la web semántica (2001). Disponible en: http://www.rediris.es/rediris/boletin/6667/ponencial 8.pdf

2. Santacruz L., Aedo I, Kloos C. ELO: Entorno para la generación, integración y reutilización de objetos de aprendizaje, (2001). Disponible en: http://www.esev.ipv.pt/3siie/actas/index.htm

3. Learnativity.org, [Internet] (2004). Disponible en: http://www.learnativity.org

4. IEEE [sede Web]. IEEE SA. (actualizado 15 de Julio de 2002). LOM (Learning Object Metadata) [44 páginas], Disponible en:

http://Itsc.ieee.org/wg 12/20020612-FinalLOM-Draft.pdf

5. Learning Objects Portal (2003). Disponible en: http://ilearn.senecac.on.ca/lop/about/about.ht $\mathrm{m}$

E-mail:Jane.herrera@gmail.com 